\title{
Estado de la cuestión sobre
}

\section{el aprendizaje y la enseñanza}

de la radiactividad en la educación secundaria

\section{Current issues in teaching and learning radioactivity in secondary education}

\author{
Javier Corbelle Cao, José Manuel Domínguez Castiñeiras \\ Departamento de Didáctica de las Ciencias Experimentales. Universidad de Santiago de Compostela. España \\ jcorbelle@edu.xunta.es, josemanuel.dominguez@usc.es
}

RESUMEN • En el presente trabajo se realiza una revisión bibliográfica sobre las dificultades de enseñanza y aprendizaje en el campo conceptual de la radiactividad. Se lleva a cabo el análisis desde diferentes perspectivas: ideas previas de los alumnos, ideas de profesores en formación, actividades de aula y propuestas didácticas. Se exploran las causas de dichas dificultades y se obtienen grandes coincidencias entre las ideas de alumnos y profesores en formación.

PALABRAS CLAVE: revisión bibliográfica; didáctica de las ciencias; radiactividad; ideas previas; pensamiento del profesorado.

ABSTRACT $\bullet$ Through the means of a literature review, this article tackles the difficulties of teaching and learning about radioactivity and its related concepts. An analysis of the issue is carried out from different angles: students' misconceptions, pre-service teachers' ideas, classroom activities and teaching proposals. Having explored the causes of such difficulties, a large number of similarities between the thoughts of students and pre-service teachers can be found.

KEYWORDS: literature review; science education; radioactivity; misconceptions; pre-service teachers' ideas. 


\section{INTRODUCCIÓN}

Los aspectos relacionados con la radiactividad suelen estar de actualidad, pues existe un debate casi permanente sobre el uso de la energía nuclear para la producción de energía eléctrica y la problemática asociada con los residuos generados. Accidentes en centrales nucleares o el uso de armamento nuclear son causa de tensión y preocupación política. Además, la radiactividad tiene numerosas aplicaciones en medicina nuclear, siendo algunas de ellas de uso tan cotidiano como la tomografía por emisión de positrones (PET) o la radioterapia.

Pero la radiactividad es un fenómeno natural con el que siempre se ha convivido (Atwood, 1992; Hutchinson y Hutchinson, 1997; Ronneau, 1990) y que está presente en nuestra vida diaria, aunque, por ejemplo, poca gente es consciente de la presencia del gas radón, de su origen y de sus peligros. Todo ello hace de la radiactividad un importante tema socio-científico.

Además, la radiactividad presenta dificultades específicas de enseñanza-aprendizaje. Como señalan Boyes y Stanisstreet (1994):

- La radiactividad es un fenómeno que no es percibido por nuestros sentidos. Tiene una naturaleza abstracta y, por tanto, es necesario representarla mediante analogías.

- Algunos aspectos de la radiactividad tienen naturaleza estocástica, como el proceso de decaimiento radiactivo o el efecto de la radiación ionizante sobre el tejido biológico.

- La influencia de los medios de comunicación que, en ocasiones, usando un lenguaje poco riguroso, puede inducir ideas erróneas.

La radiactividad es, en definitiva, un tema cargado de sentimientos como miedo o desconfianza. Un adecuado tratamiento didáctico podría ayudar a comprender y valorar los riesgos asociados con ella.

A este respecto, se ha considerado de interés presentar en este trabajo una revisión bibliográfica sobre las dificultades de enseńanza y aprendizaje en el campo conceptual de la radiactividad desde diferentes perspectivas: ideas previas de los alumnos, ideas de profesores en formación, actividades de aula y propuestas didácticas.

\section{BÚSQUEDA BIBLIOGRÁFICA. MÉTODO}

A continuación se relacionan las bases de datos y los criterios usados para la selección de la bibliografía analizada.

\section{Recuperación de la información. Fuentes documentales}

La búsqueda de la literatura se ha realizado utilizando las bases de datos que se recogen en el anexo 1 . Están especializadas en ciencias humanas y sociales y contienen amplia información sobre revistas y recursos relacionados con la educación.

\section{Análisis de la fiabilidad y validez de los artículos. Criterios de selección}

Los criterios de selección se encuentran determinados por:

a) El objetivo de la revisión: obtener una visión global sobre la problemática de la enseñanzaaprendizaje de la radiactividad desde diferentes perspectivas, a la luz de la investigación realizada hasta el momento: ideas previas de los alumnos, ideas de profesores en formación, actividades de aula y propuestas didácticas. 
b) La fiabilidad y validez de los artículos (anexo 2), teniendo en cuenta su calidad metodológica y si cumplen con los criterios de calidad científica buscada. Para ello, se han utilizado los índices de valoración de revistas científicas que se recogen en la tabla 1 .

Tabla 1.

Índices de valoración de revistas científicas

\begin{tabular}{|l|l|}
\hline \multicolumn{1}{|c|}{ Índice de impacto } & \multicolumn{1}{c|}{ Características } \\
\hline $\begin{array}{l}\text { Índice Compuesto de Difusión } \\
\text { Secundaria (ICDS) }\end{array}$ & $\begin{array}{l}\text { Indicador que mide la difusión de las revistas en bases de datos } \\
\text { científicas. El ICDS se publica mediante la base de datos Matriu } \\
\text { d'Informació per a l'Avaluació de Revistes (MIAR), elaborada } \\
\text { por el Departamento de Biblioteconomía y Documentación de la } \\
\text { Universidad de Barcelona, contiene información sobre la calidad } \\
\text { de las revistas de ciencias sociales y humanidades. }\end{array}$ \\
\hline In-RECS & $\begin{array}{l}\text { Elaborado por el grupo de investigación EC3 de Evaluación de } \\
\text { la Ciencia y de la Comunicación Científica de la Universidad de } \\
\text { Granada, calcula el factor de impacto indizando casi 200 títulos } \\
\text { de revistas españolas del ámbito de las ciencias sociales. Incluye } \\
\text { datos desde 1996 y puede consultarse por materias y por títulos } \\
\text { de revistas, artículos, autores e instituciones. Permite determinar } \\
\text { la relevancia, influencia e impacto científico de las revistas, de } \\
\text { los autores que publican en las mismas y de las instituciones a } \\
\text { las que estos se adscriben. Dejó de actualizarse en 2014. }\end{array}$ \\
\hline Journal Citation Reports (JCR) & $\begin{array}{l}\text { Lo publica y elabora anualmente Thomson-ISI y es la principal } \\
\text { herramienta utilizada para la evaluación de las revistas } \\
\text { científicas. Existe una edición para las revistas de ciencias, JCR } \\
\text { Science Edition, y otra para las de ciencias sociales, JCR Social } \\
\text { Sciences Edition. }\end{array}$ \\
\hline SCImago Journal Rank (SJR) & $\begin{array}{l}\text { Portal abierto que toma como base la información contenida en } \\
\text { la base de datos Scopus (de Elsevier). Se pueden consultar } \\
\text { indicadores de revistas científicas y países, las citaciones por } \\
\text { artículo, el índice H o el Scimago Journal Rank, un indicador de } \\
\text { visibilidad creado a partir del algoritmo de Page Rank de } \\
\text { Google. Con SJR, el área de investigación, calidad y reputación } \\
\text { de la revista científica tienen un impacto directo sobre el valor } \\
\text { de la cita. Desarrollado por SCImago. }\end{array}$ \\
\hline
\end{tabular}

\section{MARCO TEÓRICO DE REFERENCIA: CIENCIA ESCOLAR DESEABLE}

Como paso previo a la búsqueda y análisis de la bibliografía se establecen los conceptos más relevantes respecto al fenómeno de la radiactividad y se muestran las principales relaciones entre ellos, desde la perspectiva de la ciencia escolar.

En primer lugar, se debe señalar la diferencia entre los conceptos de radiactividad, fuente radiactiva y radiación. Como se ilustra en la figura 1, el término radiactividad hace referencia a un fenómeno o proceso en el transcurso del cual se emiten radiaciones ionizantes, tradicionalmente denominadas $\alpha, \beta$ y $\gamma$. Dicho fenómeno tiene su origen en la inestabilidad de ciertos núcleos atómicos que se transforman en otros diferentes, proceso denominado decaimiento radiactivo. Los tres tipos de desintegraciones radiactivas citadas constituyen los tres modos por los que dichos núcleos se transforman en núcleos más estables. Así, la desintegración tipo $\alpha$ es característica de núcleos muy pesados que necesitan perder masa, y lo hacen emitiendo núcleos de helio. La desintegración $\beta$ es característica de núcleos ricos en neutrones. Un modo de disminuir un neutrón es mediante su transformación en un protón con la consiguiente emisión de un electrón (partícula $\beta$ ). Los procesos $\gamma$ consisten en la liberación de radiación $\gamma$ cuando un nucleón (protón o neutrón) pasa de un estado cuántico de mayor energía a otro de menor energía. Por tanto, en este proceso no hay realmente una transformación de un núcleo de un elemento químico a otro de un elemento diferente. 
A este respecto, la sustancia que posee dichos núcleos se denomina sustancia o fuente radiactiva. Conviene señalar, no obstante, que una sustancia radiactiva no está compuesta únicamente por átomos radiactivos, salvo en casos excepcionales. En la figura 1, los átomos radiactivos están representados por un círculo con una cruz y el átomo en el cual decaen por un círculo blanco. Los círculos negros representan átomos estables.

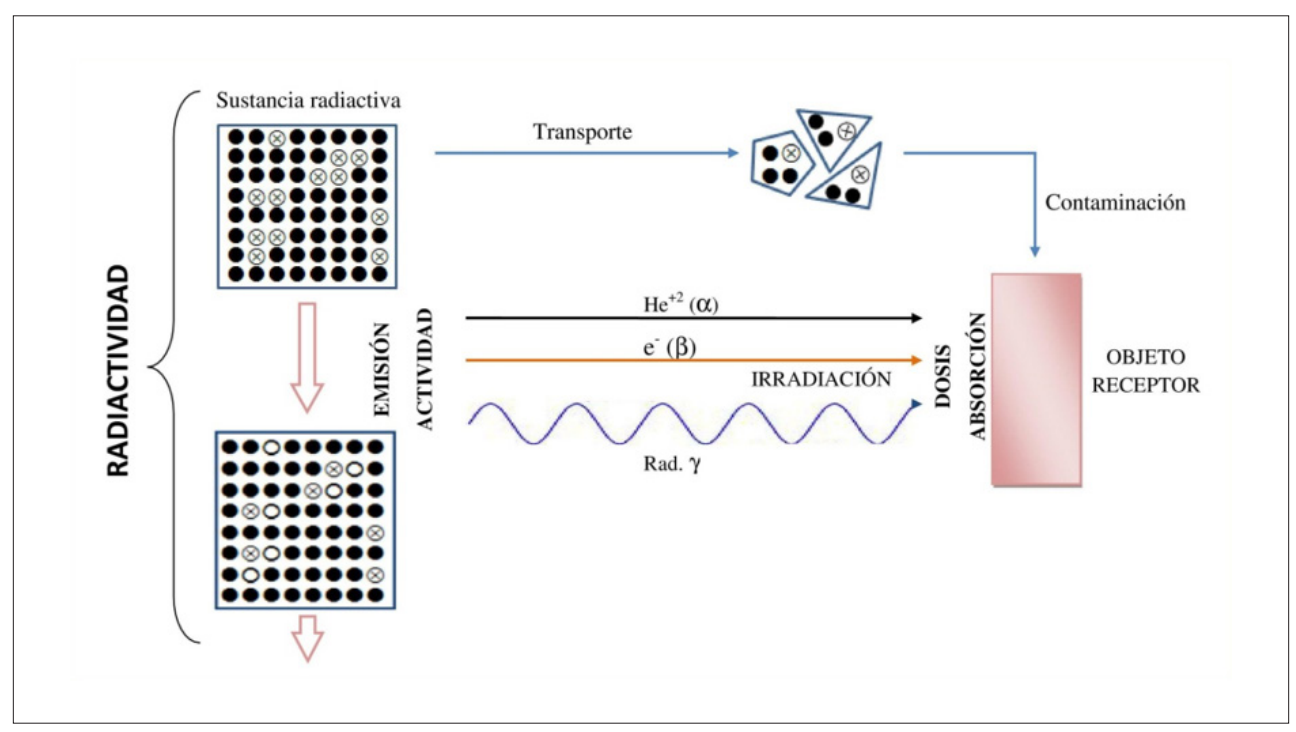

Fig. 1. Relación de conceptos clave sobre el fenómeno de la radiactividad (adaptado de Millar et al., 1990)

En segundo lugar, conviene diferenciar entre los conceptos de irradiación y contaminación. La irradiación supone que un cuerpo ha recibido radiación, en este contexto radiación ionizante. Cuando las radiaciones $\alpha$ y $\beta$ interaccionan con la materia puede producirse excitación de electrones a niveles energéticos superiores, ionización de átomos por desplazamiento de electrones de su capa electrónica más externa o ruptura de enlaces químicos por desplazamiento de electrones de enlace. En el caso de la radiación $\gamma$, se puede producir efecto Compton, efecto fotoeléctrico o formación de pares electrónpositrón, dependiendo de su energía. En cambio, es necesaria la presencia de sustancias radiactivas en un cuerpo para que exista contaminación. En el caso de las personas dicha contaminación puede producirse por deposición, inhalación, ingestión o inyección de sustancias radiactivas.

El concepto de actividad hace referencia a las sustancias radiactivas (figura 1), en concreto al ritmo o velocidad con que están decayendo los núcleos responsables del fenómeno radiactivo, según la expresión:

$$
\mathrm{A}(\mathrm{t})=-\frac{\mathrm{dN}(\mathrm{t})}{\mathrm{dt}}=\lambda \cdot \mathrm{N}(\mathrm{t})
$$

[ecuación 1]

Donde $A(t)$ es la actividad de la muestra en el instante $t, N(t)$ es el número de núcleos radiactivos presentes en ese momento y $\lambda$ es una constante radiactiva característica de cada radioisótopo. La unidad en el sistema internacional es el Becquerel (Bq). Una actividad de $1 \mathrm{~Bq}$ es una desintegración por segundo. La actividad de una muestra dada disminuye exponencialmente con el tiempo de acuerdo con la función:

$$
\mathrm{A}(\mathrm{t})=\mathrm{A}_{0} \mathrm{e}^{-\lambda \mathrm{t}}
$$

[ecuación 2] 
Donde $\mathrm{A}_{0}$ es la actividad inicial de dicha muestra. Como se aprecia en la ecuación 1 la actividad depende del tipo de núcleo y de su número en ese momento.

En cambio, el concepto de dosis hace referencia a la cantidad de radiación recibida por un cuerpo. Por ello, es este concepto el usado para estimar la peligrosidad o el riesgo asociado al fenómeno de la radiactividad. Cuando la radiación ionizante interacciona con la materia produce alteraciones, como se ha señalado anteriormente, que van a depender de la cantidad y naturaleza de la radiación recibida. Las medidas de protección irán, por tanto, encaminadas a reducir la dosis de radiación ionizante recibida. Son tres los factores que hay que tener en cuenta: tiempo de exposición, cuanto menor sea menor será la dosis recibida; distancia, cuanto mayor sea menor será la dosis recibida; y blindaje, que debe impedir que la radiación emitida por la sustancia radiactiva lo atraviese.

Otro concepto importante es el periodo de semidesintegración. El decaimiento de un núcleo dado es un proceso espontáneo y aleatorio. No es posible saber cuándo un núcleo va a decaer. Por tanto, el estudio de estos procesos se realiza desde un punto de vista estadístico. Desde esta perspectiva, se define el periodo de semidesintegración como el tiempo necesario para que decaigan la mitad de los núcleos radiactivos inicialmente presentes en una muestra. Esto no significa que la muestra pierda átomos sino que está teniendo lugar una transformación de núcleos de un elemento químico, en núcleos de otro elemento químico.

Finalmente, se debe añadir que la radiactividad es un fenómeno natural al que estamos continuamente expuestos. En la tabla 2 y la figura 2 se muestran las principales fuentes naturales de radiación ionizante, así como las dosis medias recibidas a nivel mundial.

Tabla 2

Fuentes radiactivas naturales y dosis recibidas

\begin{tabular}{|c|c|c|}
\hline $\begin{array}{l}\text { Fuentes naturales de } \\
\text { radiación ionizante }\end{array}$ & Principales partículas responsables & $\begin{array}{l}\text { Dosis efectiva } \\
\text { (mSv/año) })^{(*)}\end{array}$ \\
\hline Radiación cósmica & $\begin{array}{l}\text { Muones (representan } \approx 80 \% \text { ), fotones, } \\
\text { electrones, neutrones, protones y } \\
\text { otras partículas cargadas. }\end{array}$ & 0,38 \\
\hline $\begin{array}{c}\text { Radiación cosmogénica } \\
\text { (núcleos originados por los rayos } \\
\text { cósmicos en la atmósfera) }\end{array}$ & $\begin{array}{l}{ }^{14} \mathrm{C}(12 \mu \text { Sv/año }) ;{ }^{22} \mathrm{Na}(0,15 \mu \text { Sv/año }) ; \\
{ }^{7} \operatorname{Be}(0,03 \mu \text { Sv/año }) ;{ }^{3} \mathrm{H}(0,01 \mu \text { Sv/año })\end{array}$ & 0,01 \\
\hline $\begin{array}{l}\text { Radiación terrestre } \\
\text { (Radiación gamma procedente del } \\
\text { suelo y materiales de construcción) }\end{array}$ & $\begin{array}{l}{ }^{40} \mathrm{~K} \\
\text { Productos de las series de }{ }^{238} \mathrm{U} \mathrm{y}^{232} \mathrm{Th}\end{array}$ & 0,48 \\
\hline $\begin{array}{l}\text { Inhalación } \\
\text { (Gas Radón o partículas de polvo en } \\
\text { el aire que contienen elementos de } \\
\text { las series del }{ }^{238} \mathrm{Uy}^{232} \mathrm{Th} \text { ) }\end{array}$ & $\begin{array}{l}\text { Series del }{ }^{238} \mathrm{U} \mathrm{y}^{232} \mathrm{Th} \\
{ }^{222} \mathrm{Rn} \text { (Radón) } \\
{ }^{220} \mathrm{Rn} \text { (Torón) }\end{array}$ & $\begin{array}{c}0,006 \\
1,15 \\
0,1\end{array}$ \\
\hline $\begin{array}{c}\text { Ingestión } \\
\text { (Bebidas y alimentos que contienen) }\end{array}$ & 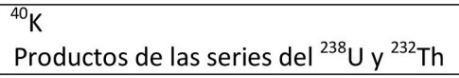 & $\begin{array}{l}0,17 \\
0,12\end{array}$ \\
\hline Total & & 2,4 \\
\hline
\end{tabular}

${ }^{(*)}$ Dosis efectiva recibida por el ser humano procedente de fuentes naturales, al nivel del mar. Valores medios mundiales.

Fuente: Elaboración propia a partir de UNSCEAR (2010) 


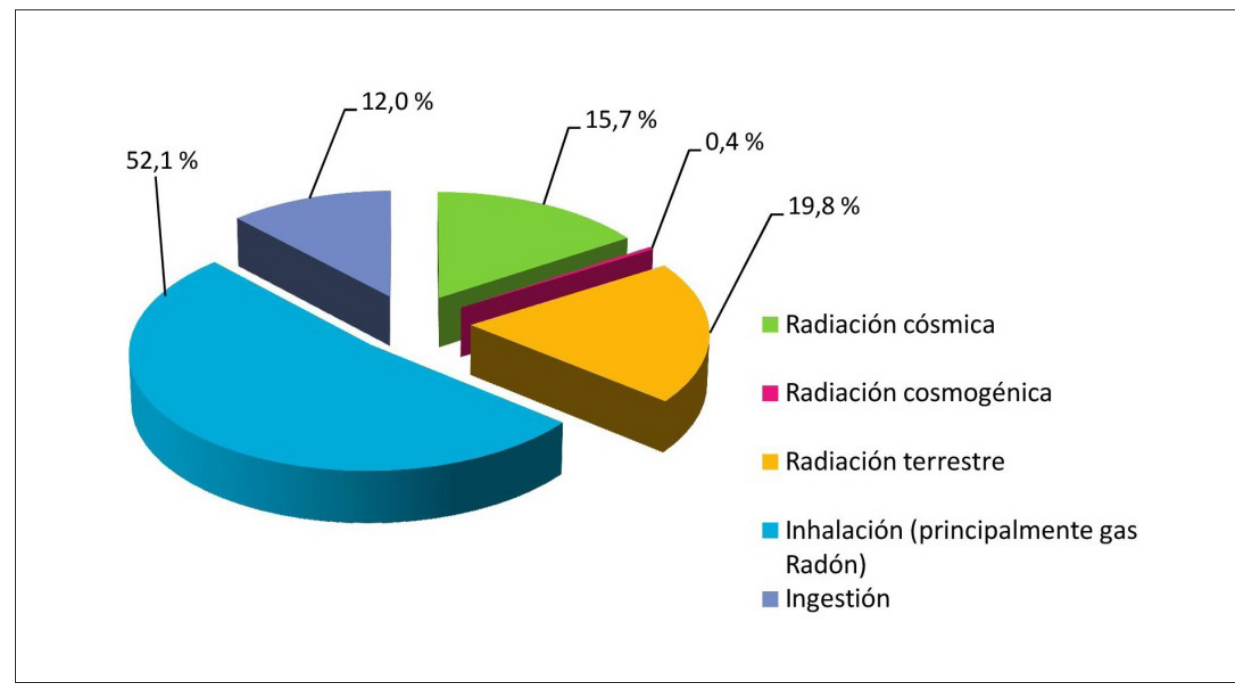

Fig. 2. Porcentajes de dosis recibidas procedentes de fuentes radiactivas naturales. Valores medios mundiales. Fuente: Elaboración propia a partir de UNSCEAR (2010)

\section{REVISIÓN BIBLIOGRÁFICA. RESULTADOS}

De acuerdo con la metodología señalada, se realiza la revisión bibliográfica desde las tres perspectivas siguientes:

- Ideas previas de los alumnos

- Ideas de profesores en formación

- Actividades de aula y propuestas didácticas

En los apartados A y B se han establecido una serie de categorías para realizar el análisis y la obtención de conclusiones. Hay que señalar, no obstante, que dichas categorías han sido deducidas directamente de los propios trabajos de investigación, si bien algunas de ellas han sido reformuladas o agrupadas para facilitar dicho análisis. Dado que dichas categorías vienen determinadas por los aspectos tratados en los diferentes estudios objeto de esta revisión, no existe una coincidencia total entre ellas en dichos apartados.

\section{A. Ideas previas de los alumnos}

Este análisis se realiza desde una doble perspectiva: desde un punto de vista macroscópico, más centrado en el aspecto fenomenológico, y desde un plano que considera el proceso radiactivo a nivel atómico y nuclear.

\section{Perspectiva macroscópica}

- Sobre los términos radiactividadl fuente radiactival radiación

Se ha encontrado que, en general, el alumnado tanto de enseñanza secundaria (Millar et al., 1990; Millar y Singh, 1996) como de primer ańo de universidad (Prather y Harrington, 2001) hace uso indiferenciado y generalizado de los términos radiación, fuente radiactiva y radiactividad. Por ejemplo, hablan de «la cantidad de radiactividad procedente de la central nuclear» o de 
que «la radiación» ha sido llevada desde Chernóbil hasta Gran Bretaña por el viento (Millar y Singh, 1996), e incluso de «radiación radiactiva, ondas radiactivas o partículas radiactivas», para describir la radiación emitida (Prather y Harrington, 2001).

- Sobre las fuentes radiactivas

Boyes y Stanisstreet (1994), en un amplio estudio realizado con 1.365 alumnos entre 11 y 16 años, observaron que la mayoría (80\%) cree que la radiactividad es un fenómeno que no se percibe como natural, sino como algo producido por el hombre y asociado con la alta tecnología: centrales de energía nuclear. Solo un 30\% de estudiantes afirmaron que el espacio exterior, las rocas o los gases procedentes del suelo eran fuentes radiactivas; en este último caso, consideraban la radiactividad como un gas. Alsop (2001) encuentra este último problema entre el alumnado universitario. Desde la perspectiva microscópica, Prather y Harrington (2001) indican que alumnos de primer año de universidad conciben las fuentes radiactivas como formadas únicamente por átomos radiactivos.

- Sobre los términos irradiación/ contaminación

Los trabajos de Eijkelhof (1989), Henriksen y Jorde (2001) y Millar et al. (1990) ponen de manifiesto que el alumnado de educación secundaria confunde irradiación y contaminación, considerando que la radiación interacciona con la materia y es almacenada en el objeto irradiado, pudiendo ser liberada más tarde. Lo mismo encuentran Prather y Harrington (2001): en el caso de un paciente que recibe radiación sobre un tumor y otro al que se le inyecta una sustancia radiactiva en el torrente sanguíneo, pocos alumnos establecen correctamente que solo en el segundo caso hay contaminación. Resultados similares fueron obtenidos por Millar y Singh (1996) al analizar las respuestas de un ejercicio sobre dos noticias de prensa.

- Sobre los modos de propagación de la radiación emitida

El alumnado de enseñanza secundaria afirma que la radiación emitida por una sustancia radiactiva se propaga mediante ondas, partículas (Boyes y Stanisstreet, 1994) o consiste en ondas y partículas (Gutiérrez et al., 2000). Pero, además, un número significativo cree que se propaga como calor o como una nube de gas, confundiendo, en este último caso, sustancia radiactiva con radiación, como se mencionó anteriormente. No se percibe que la radiación tiene un alcance limitado y local, mientras que el material radiactivo o las sustancias contaminadas con material radiactivo pueden viajar grandes distancias, teniendo así un mayor alcance. Millar y Singh (1996) encuentran esta misma confusión cuando el alumnado intenta explicar los efectos limitados de una fuente radiactiva en el laboratorio y el largo alcance de las consecuencias del accidente en la central nuclear de Chernóbil.

- Sobre los efectos de la radiactividad y medidas protectoras

La radiactividad se asocia en la mayoría de los casos con peligro, pero afectando de manera diferente a la materia inerte y a los seres vivos. Estos últimos se perciben como especialmente vulnerables al fenómeno radiactivo.

En el marco del proyecto PLON (Eijkelhof, 1990; Eijkelhof y Lijnse, 1988), se obtuvieron las siguientes creencias de estudiantes de secundaria respecto a la radiación ionizante, desde la perspectiva de la evaluación del riesgo (Eijkelhof, 1989; Eijkelhof et al., 1989):

a) Sobre la radiación ionizante:

- La radiación es siempre peligrosa. Los alumnos que tienen esta creencia tienden a razonar que la radiación no desaparece sino que se acumula en los objetos. Además, no tienen en cuenta la relación dosis-efecto, considerando que cualquier dosis recibida será peligrosa.

- Los riesgos de la radiación son limitados, es decir, la radiación podría ir disminuyendo hasta cierto nivel por debajo del cual no es peligrosa. 
b) Sobre la percepción del riesgo y su relación con el contexto:

- Si la radiación se usa con un fin determinado, no es percibida como peligrosa (aplicaciones médicas frente a residuos radiactivos).

- Si la radiación es natural (radiación natural de fondo), no es percibida como peligrosa en la mayoría de los casos. Esto mismo se encontró en otras investigaciones: el mineral de uranio (natural) es percibido como menos peligroso que el uranio usado en una central nuclear (artificial) (De Posada y Prieto, 1990); o las fuentes radiactivas artificiales se perciben como más peligrosas porque en ellas la radiactividad «estaría más concentrada» (Alsop, 2001).

- Si la radiación se asocia con situaciones objeto de información reiterada en los medios de comunicación (residuos radiactivos, accidentes en centrales nucleares), es percibida como peligrosa.

El modo en que las sustancias radiactivas afectan a la materia inerte depende del material. Así, los objetos metálicos tendrían la capacidad de atraer la radiación, sugiriendo una asociación entre radiactividad y magnetismo (Alsop, 2001). El plástico, en cambio, se percibe como impenetrable a la radiación, lo que podría ser una consecuencia de las imágenes vistas en televisión cuando ocurre algún accidente en una central nuclear o científicos trabajando con materiales radiactivos. Asimismo, algunos alumnos asocian la radiactividad con problemas medioambientales como la capa de ozono y el efecto invernadero (Boyes y Stanisstreet, 1994).

\section{Perspectiva atómico-nuclear}

La radiactividad es un proceso cuyo origen reside en el comportamiento del núcleo atómico y a este nivel ha de ser interpretada. Teniendo en cuenta la dificultad que los alumnos muestran para interpretar adecuadamente la naturaleza atómica de la materia (por ejemplo, Nussbaum, 1989; Harrison y Treagust, 1996), cabe pensar que de igual manera la tengan para interpretar el fenómeno radiactivo desde la perspectiva nuclear. Millar et al. (1990) hacen referencia a investigaciones llevadas a cabo por Kees Klaassen con alumnos holandeses de enseñanza secundaria, indicando las dificultades que tienen para explicar el fenómeno radiactivo a nivel atómico. Y Boyes y Stanisstreet (1994) encontraron que algunos alumnos entre 11 y 16 años pensaban que el accidente de Chernóbil había sido un accidente químico, interpretando estos autores que los procesos nucleares no son muy diferentes de los procesos químicos, a los ojos de estos alumnos.

Prather (2005), en un estudio con alumnos de college matriculados en cursos introductorios de Física, sostiene que muchas de las ideas erróneas que los alumnos tienen sobre el fenómeno de la radiactividad, como por ejemplo la no diferenciación entre irradiación y contaminación, se debe a una falta de comprensión del comportamiento del átomo durante dicho proceso. De este estudio se derivaron los resultados que se comentan a continuación.

- Sobre el átomo radiactivo

Para justificar que un átomo es radiactivo, participaron 128 alumnos. Solo el 20\% hizo referencia exclusivamente a las partículas presentes en el núcleo o a la estructura de este. Algo más del $50 \%$ incluyó los electrones de valencia para este fin. Solo el 60\% de ellos hizo un dibujo correcto del átomo, incluyendo la localización y la carga correcta de sus partículas constituyentes.

Algunos de los resultados encontrados por Prather (op. cit.) coinciden con los de Nakiboğlu y Tekin (2006) en un estudio realizado con 157 alumnos de secundaria (15-16 ańos), después de haber recibido docencia sobre una unidad de química nuclear. Las dos opciones mayoritariamente elegidas fueron que la inestabilidad del núcleo de los átomos era debida a la proporción 
entre el número atómico y su número másico (52\%) y tener completa su capa de valencia $(26 \%)$.

- Sobre el decaimiento radiactivo y periodo de semidesintegración

Para estudiar el conocimiento del alumnado sobre el proceso de decaimiento, Prather (op. cit.) analizó las respuestas de 277 estudiantes -entre los que se encontraban, además, 19 alumnos de bachillerato- que agrupó en las siguientes categorías:

- Un 45\% solo hace uso de electrones de valencia.

- Un 30\% solo hace uso de neutrones, protones o el núcleo.

- Un 20\% elabora respuestas mixtas, implicando electrones de valencia y el núcleo.

Para conocer las ideas sobre el periodo de semidesintegración se analizaron las respuestas 143 alumnos (Prather, op. cit.). Parece deducirse que piensan que, después de un tiempo igual a un periodo de semidesintegración, la mitad de la sustancia radiactiva simplemente desaparece, tal vez pensando que una sustancia radiactiva está compuesta únicamente por átomos radiactivos. Un ejemplo de respuesta sería: «Es el tiempo que le lleva a un objeto convertirse en la mitad de lo que era antes».

Por último, señalar que -después de que 65 alumnos participantes en este estudio (Prather, op. cit.) recibiesen tres horas de clase tradicional, dos horas de clase de resolución de problemas y dos horas de prácticas de laboratorio- el 59\% de ellos continuaban creyendo que la masa y/o el volumen se reduce a la mitad después de un tiempo igual a un periodo de semidesintegración.

\section{B. Ideas de los profesores en formación}

Solo se han encontrado tres trabajos (Aubrecht y Torick, 2000; Colclough et al. 2011; Ergül, 2012) en los que, de forma específica, se investigan las ideas de profesores en formación sobre el campo conceptual de la radiactividad. Al igual que se hizo para el alumnado, se han clasificado las ideas de los profesores en formación en las siguientes categorías.

\section{Perspectiva macroscópica}

- Sobre las fuentes radiactivas

Tanto Aubrecht y Torick (2000) como Ergül (2012) obtienen que un número significativo de profesores en formación relacionan las fuentes radiactivas con la actividad humana o con la tecnología (hornos microondas, laboratorio de rayos $\mathrm{X}$, cables eléctricos de alta tensión), sin ser conscientes de la continua exposición a las fuentes radiactivas naturales y confundiendo fuentes de radiación ionizante con fuentes de radiación no ionizante.

Existe un debate sobre hasta qué punto la formación previa influye en el conocimiento en esta área. Por ejemplo, Ergül (2012) no encuentra diferencia entre el profesorado en formación de educación primaria y el de Ciencia, Tecnología y Física. Colclough et al. (2011), sin embargo, obtienen que el número de respuestas correctas de los graduados en Física, respecto a la identificación de fuentes de radiación natural, es mucho mayor que los de Química, y las de estos superiores a los de Biología. Los de Historia reflejan el número más bajo de respuestas correctas. Por otra parte, las fuentes radiactivas son percibidas como formadas íntegramente por átomos radiactivos (Aubrecht y Torick, 2000), considerando que disminuye la masa y/o el volumen a la mitad después de un tiempo igual a un periodo de semidesintegración, resultado obtenido también por Prather (2005) con alumnos de primer año de universidad. 
- Sobre los términos irradiación/contaminación

Colclough et al. (2011) obtienen que la mayoría de los profesores en formación, incluyendo a los graduados en Física, sostienen que una sustancia irradiada se convierte en radiactiva. Sugieren que podría haber dos corrientes de razonamiento:

- Sujetos que piensan que al irradiar un objeto se produce una fuente secundaria de radiación (radiación inducida). Por ejemplo, ante un espejo irradiado con una fuente de Ra-226, uno de los graduados en Física dice: «estará ligeramente radiado [el espejo] [...] partes del soporte de plata están ligeramente ionizadas e irradiadas así que [...] liberan más radiación».

- Sujetos que muestran una confusión entre irradiación y contaminación. Ante un trozo de carne irradiada uno de los graduados en Historia dice: "Yo no me expondría a un potencial riesgo (al comerla). Creo que todavía habrá algo de radiación absorbida en la carne». Parece deducirse de esta afirmación que el contaminante es la propia radiación que quedaría almacenada.

- Sobre los efectos de la radiactividad y medidas protectoras

Colclough et al. (2011) encuentran que la enseñanza formal de los profesores en formación en el campo de la radiactividad influye en una mayor aceptabilidad del riesgo y en que el lenguaje usado sea menos sensacionalista. Así, los graduados en Física son los más dispuestos a usar un espejo previamente irradiado, siguiendo en orden descendente los graduados en Química, Biología e Historia. Sin embargo, en los cuatro grupos se observó una menor aceptación del riesgo cuando se trataba de tejido vivo: por ejemplo, comer carne irradiada o usar un reloj cuyo dial contiene sustancias radiactivas. Esto concuerda con lo observado por Alsop (2001) en alumnos universitarios (los organismos vivos son vistos como particularmente vulnerables a la radiactividad).

Cuando se evalúan riesgos asociados con la radiactividad y la radiación ionizante, normalmente se suelen considerar tres elementos clave: tiempo de exposición, distancia y blindaje, ya que son los elementos fundamentales que determinan la dosis absorbida. También se suele hacer referencia al nivel aceptable de exposición, comparándolo con la dosis absorbida por radiación natural. Colclough et al. (2011), obtienen que mientras que los graduados en física fueron los que más factores consideraron, seguidos por biólogos y químicos, los graduados en historia apenas usaron alguno. Sin embargo, Ergül (2012), al analizar las cuestiones relativas a los efectos de la radiación sobre los organismos vivos, no encuentra diferencias entre los tres grupos, excepto en la cuestión "Los efectos de la radiación en organismos ocurren de forma inmediata», donde el número de respuestas correctas de los graduados en Física es significativamente mayor.

Asimismo, Aubrecht y Torick (2000) obtuvieron que, en general, los profesores en formación no eran conscientes de que diferentes tipos de procesos radiactivos (alfa, beta o gamma) requerían diferentes tipos de protección. El equipo de protección que eligen depende de su experiencia previa, bien procedente de los medios de comunicación (noticias sobre centrales nucleares, dibujos animados, etc.), bien de la visita al hospital, u otras. Por ejemplo, uno de ellos hace referencia a una bata de plomo porque es «lo que ellos usan cuando haces una radiografía de rayos X». 
Perspectiva atómico-nuclear

- Sobre la interacción de la radiación con la materia

En general, se observaron bastantes deficiencias ya que, en muchos casos, no se hace uso de una interpretación de este fenómeno a nivel atómico. Los resultados son mejores, cuanto mayor ha sido la enseñanza formal en el área de Física.

Por ejemplo, en el estudio de Colclough et al. (2011), diez de los doce futuros profesores entrevistados interpretaban la absorción en términos de la acción bloqueante producida por diferentes materiales. La capacidad bloqueante del material depende de sus propiedades macroscópicas: la densidad o la superficie del material. Algunos de los que hicieron uso del concepto de ionización también mostraron deficiencias. Por ejemplo, un graduado en química afirma que las partículas alfa ionizaban el átomo porque extraen electrones del núcleo atómico; y un graduado en biología interpreta incorrectamente la ionización en términos de excitación del átomo. Asimismo, siete de doce graduados consideraron erróneamente que la radiación $\gamma$ posee un mayor poder de penetración que las partículas $\alpha$ y $\beta$, simplemente porque es "más energética».

- Sobre el decaimiento radiactivo y periodo de semidesintegración

Aubrecht y Torick (2000), encuentran que muchos profesores en formación piensan que el proceso de decaimiento estará influenciado por la posición de los átomos radiactivos en la muestra. O que se verá afectado por factores externos al núcleo, como la temperatura o el estado físico de la sustancia radiactiva. A mayor temperatura más rápido sería el proceso de decaimiento. Las sustancias gaseosas tendrían una mayor actividad que las sustancias líquidas o sólidas.

Estos mismos autores ponen de manifiesto que muchos de ellos consideran que cuanto mayor es el periodo de semidesintegración o la vida media, más tiempo durarán los átomos radiactivos y, por tanto, más daño causarán. Por ejemplo, al relacionar el carbono-14 ( $\mathrm{T}=5730$ años) con el uranio-235 ( $\mathrm{T}=700$ millones de años), uno de los profesores en formación argumenta que el uranio «causaría más daño porque está viviendo más tiempo y está liberando más ondas radiactivas que los otros». Parece desprenderse de lo anterior que la actividad de una sustancia radiactiva permanece constante con el tiempo, coexistiendo, además, la confusión entre actividad y dosis recibida.

\section{Actividades de aula y propuestas didácticas}

Existen muchas actividades de aula sobre este tema aunque concentradas fundamentalmente en dos aspectos: las que ponen de manifiesto la radiactividad natural y aquellas que ilustran el proceso de decaimiento radiactivo. Asimismo, hay trabajos que se pueden englobar en el marco del movimiento ciencia-tecnología-sociedad (CTS) o asuntos sociocientíficos. Por el contrario, como se indicará más abajo, apenas se han encontrado propuestas didácticas completas.

- Actividades sobre fuentes radiactivas naturales

Se trata de trabajos prácticos que, usando un contador Geiger-Müller, evalúan diferentes aspectos de la radiactividad natural. Algunos de ellos consisten en medir la radiación emitida por productos comunes que contienen sustancias radiactivas (Couch y Vaughn, 1995; Lapp, 2010; Peplow, 1999; Russo, 1999; Whitcher, 2011). Otras son actividades de campo en las cuales se mide la dosis de radiación natural recibida en diferentes escenarios (Al-Azmi et al., 2013; Anjos et al., 2008). Existen trabajos que ilustran cómo concentrar fuentes radiactivas y así obtener medidas de actividad que se puedan diferenciar suficientemente de la actividad natural. Por ejemplo, Herrmann (1974) explica el procedimiento para obtener radio a partir de relojes antiguos; otros autores (Cookson, 1999; Whitcher, 2011) muestran cómo concentrar radón presente en 
el ambiente usando un globo cargado eléctricamente mediante fricción; y Al-Azmi et al. (2012) cómo concentrar el mismo gas adsorbiéndolo sobre carbón activo. Asimismo, Gipps (1995) usa sales de potasio para, midiendo las emisiones del K-40 presente en ellas, establecer un método de análisis de este en sustancias sólidas.

También hay actividades centradas en la radiación emitida por el cuerpo humano. Así, Leenson (2006) ilustra, mediante un ejercicio de lápiz y papel, hasta qué punto nosotros mismos somos radiactivos. Y Myers (2012) mide la radiación emitida por su propio cuerpo después de haber sido inyectado con ${ }^{99 \mathrm{~m}} \mathrm{Tc}$ al haber sido diagnosticado de un cáncer de próstata. Resultan interesantes sus reflexiones sobre las relaciones intensidad de radiación-distancia e intensidad de radiación-tiempo a partir de sus propias mediciones.

Lo interesante de todas estas actividades reside en que contribuyen a resolver una deficiencia que presentan tanto alumnos como profesores en formación, ya indicada en los apartados A y B de este trabajo: no ser conscientes de nuestra continua exposición a fuentes radiactivas de origen natural, o que incluso nosotros mismos somos un poco radiactivos.

- Actividades sobre el decaimiento radiactivo y el periodo de semidesintegración

Otro grupo de actividades ilustran el proceso de decaimiento radiactivo a través de analogías (Bindel, 1988; Christian, 1988; Hughes y Zalts, 2000; Kowalski, 1981; McGeachy, 1988; Shultz, 1997) o mediante simulaciones de ordenador (Jesse, 2003; Franco, 2015; Fendt, 2015). Se trata de actividades encaminadas a ilustrar la función exponencial típica de un proceso de decaimiento radiactivo, el concepto de periodo de semidesintegración, la datación radiactiva y el carácter aleatorio de la desintegración radiactiva.

No obstante, debemos advertir sobre dos problemas que pueden presentar estas actividades. En algunas de ellas se puede reforzar la idea de que cuando un átomo se desintegra simplemente desaparece (Kowalski, 1981; Shultz, 1997), o que una sustancia radiactiva está formada únicamente por átomos radiactivos (Franco, 2015; Fendt, 2015). Ambas ideas son erróneas y han sido encontradas, como se indicó, tanto en alumnos como profesores en formación.

También existen experiencias prácticas. Por ejemplo, Mak (1999) utiliza una muestra de KCl para medir el periodo de semidesintegración del ${ }^{40} \mathrm{~K}$, y Brown (2014) hace uso de dos fuentes de ${ }^{60} \mathrm{Co}$ fabricadas en diferentes años para mostrar un ejemplo de datación. Estas dos últimas actividades presentan varias ventajas frente a las anteriores: se trata de actividades prácticas realizadas en el laboratorio, acercan al alumnado a una fuente radiactiva natural, el ${ }^{40} \mathrm{~K}$ presente en la sal de $\mathrm{KCl}$, y otra artificial, el ${ }^{60} \mathrm{Co}$. Además, el trabajo realizado en esta última actividad es fácilmente transferible a la datación por ${ }^{14} \mathrm{C}$.

Finalmente, hay trabajos que muestran cómo nos podemos apoyar en las nuevas tecnologías. Kuhn et al. (2014) usan smarthphones y tablets para estudiar la disminución en la intensidad de la radiación emitida con el cuadrado de la distancia, la absorción de la radiación por diferentes materiales y el periodo de semidesintegración, usando una fuente comercial para centros de enseñanza de ${ }^{90}$ Sr. Y Jona y Vondracek (2013) señalan la posibilidad de realizar experimentos remotos.

- La radiactividad como asunto socio-científico

En este apartado se incluyen estudios que tratan aspectos relacionados con la radiactividad desde el punto de vista del movimiento ciencia-tecnología-sociedad o bajo la perspectiva de lo que se ha denominado asuntos sociocientíficos controvertidos (Kolstø, 2001; Zeidler et al., 2005). Se trata de trabajos muy variados, la mayoría de los cuales usan como tema la energía nuclear. Por ejemplo, Wu y Tsai (2007) analizan los modos de razonamiento informal en relación al uso de la energía nuclear y señalan la dificultad que tienen los estudiantes para crear contraargumentos. Jho et al. (2014) estudian los factores que intervienen en la toma de decisiones 
respecto al uso de dicha energía, encontrando que la actitud positiva de los alumnos hacia el uso de la energía nuclear apenas mejoraba con los conocimientos adquiridos no mostrando, estos, influencia sobre la toma de decisiones. Y Showers y Shrigley (1995) toman la energía nuclear como ejemplo de un contenido CTS para mostrar que el simple aprendizaje de los contenidos no genera actitudes positivas hacia dicha energía sino que, para ello, se han de diseñar actividades persuasivas específicas.

García-Carmona y Criado (2008) analizan el enfoque CTS que recibe la energía nuclear en los libros de texto de ESO. Concluyen que, aunque el tratamiento es desigual en la muestra analizada, se observan numerosas carencias como la ausencia en un número importante de textos de los aspectos socioeconómicos y políticos relacionados con la energía nuclear, o la presentación de estos contenidos desligados del proceso de construcción y desarrollo de la ciencia nuclear en su contexto histórico y como resultado de un trabajo en equipo, entre otras.

Por otra parte, Esteban y Pérez-Esteban (2012) muestran un ejemplo del uso de una noticia de prensa, el fallecimiento del ex-espía ruso Alexis Litvinenko envenenado con polonio, para introducir conceptos sobre radiactividad.

- Estudios en contextos no formales

Henriksen y Jorde (2001) utilizan como recurso didáctico la visita a un museo para estudiar la comprensión adquirida por alumnos de 16 ańos sobre un tema de medioambiente, en el que se incluye un apartado sobre radiación ionizante y salud. Concluyen que el $25 \%$ de ellos mantiene sus ideas previas y presentan dificultades para aplicar los conocimientos adquiridos a aspectos de la vida diaria. Además, se comprobó que el lenguaje usado por los alumnos pasó de ser, en el pretest, más sensacionalista y con cierta carga emotiva a ser, en el postest, más sensato, menos personal y con un mayor uso de términos científicos. Alsop (2001) compara el grado de conocimiento y objetividad sobre radiactividad de un grupo de estudiantes universitarios que cursan carreras no científicas y viven en un área con altos niveles de gas radón con otro grupo que no lo hace. No encuentra diferencia entre ambos grupos salvo que el primero muestra un mayor conocimiento sobre los aspectos prácticos del día a día de vivir en un área con altos niveles de dicho gas. Alsop y Watts (1997) exploran la idoneidad de un modelo ampliado de cambio conceptual, usando como marco el aprendizaje informal sobre radiactividad con adultos. Estos autores estudian también hasta qué punto el aspecto afectivo puede influir sobre el aprendizaje en este campo conceptual (Alsop, 2000; Alsop et al., 1998; Alsop y Watts, 2000).

- Propuestas didácticas

Al realizar esta revisión bibliográfica, se pretendía encontrar trabajos que tratasen la enseñanza y aprendizaje de la radiactividad y radiación ionizante de un modo global y basados en los resultados de la investigación didáctica puesto que, como se ha señalado (Cooper et al., 2003) y al igual que se ha puesto de manifiesto en otras áreas de la ciencia, la enseñanza tradicional no logra modificar las ideas previas de los alumnos. Desde esta perspectiva, se ha de señalar una secuencia didáctica (Baker et al., 1973; Hawkins y Phelps, 1975) cuyo objetivo es que los estudiantes adquieran los conceptos clave sobre radiactividad mediante el diseño de una serie de actividades experimentales. Más reciente es la propuesta de Millar et al. (1990). Estos autores exponen una secuencia de enseñanza compuesta de cuatro bloques sucesivos: una orientación fenomenológica, un tratamiento macroscópico cualitativo, un tratamiento macroscópico cuantitativo y un tratamiento microscópico. Dicha secuencia está prevista para alumnos de 14-16 ańos y tendría una duración de diez sesiones lectivas de 70 minutos cada una, pudiendo impartirse en uno o dos cursos académicos. 


\section{CONCLUSIONES}

De los estudios realizados con alumnos, se observa gran número de ideas o concepciones que no se corresponden con las deseables desde el punto de vista de la ciencia escolar. Cabe preguntarse de dónde proceden y cómo han sido adquiridas. Pozo (1996) establece una clasificación de las concepciones alternativas de los alumnos, atendiendo a su origen, en tres grupos: sensoriales o concepciones espontáneas, culturales o concepciones sociales y de origen educativo o concepciones escolares. Teniendo en cuenta que la radiactividad es un fenómeno que no es percibido por nuestros sentidos, cabe esperar que dichas concepciones procedan del ámbito social y educativo.

Con relación al ámbito social, los medios de comunicación podrían representar un papel importante debido al uso de un lenguaje inadecuado, impreciso o simplemente erróneo cuando tratan temas relacionados con los fenómenos radiactivos. A este respecto conviene señalar la importancia fundamental del lenguaje para hacer ciencia. «Porque el lenguaje no es solo vocabulario y gramática: el lenguaje es un sistema de recursos para construir significados» (Lemke, 1997: 12).

El uso indiscriminado e indiferenciado de los términos radiación, radiactividad y fuente radiactiva detectado en el alumnado parece tener, al menos, como uno de sus orígenes los medios de comunicación, la interacción social y el uso que se hace del lenguaje. Asimismo, las dificultades observadas para diferenciar los términos irradiación y contaminación, o la absorción de la radiación por la materia, podrían estar muy influenciadas por este aspecto. Titulares como el siguiente ciertamente no hacen más que dificultar la correcta comprensión de conceptos como los señalados: «Los vascos tendrán en su historial clínico la radiación acumulada» (La Voz de Galicia, 14 de febrero de 2012).

Del mismo modo, cuando el alumnado opina sobre medidas protectoras contra la radiación ionizante surgen ideas extraídas del ámbito social (experiencias cotidianas como visitas al hospital, imágenes de televisión, etc.). Los medios de comunicación también pueden actuar como potenciadores de la idea de los efectos nocivos de la radiactividad sobre los seres vivos.

Con relación al ámbito educativo, se han de tener en cuenta las dificultades que poseen los alumnos para interpretar el comportamiento de la materia a nivel atómico-molecular. Ejemplos de estudios al respecto ya han sido mencionados en este trabajo. Estas dificultades se amplían ahora al nivel nuclear. Cabe preguntarse hasta qué punto la enseñanza (profesores, libros de texto y otros recursos didácticos como simulaciones por ordenador) puede reforzar algunas de estas dificultades. Respecto a ello, lo que se constata en este trabajo es un gran número de coincidencias entre ideas de alumnos y profesores en formación. Un resumen de ellas se muestra en la tabla 3.

En cuanto a los futuros profesores queremos comentar dos cuestiones: la primera, que existe una alta probabilidad de que un futuro profesor que muestra ideas que están en desacuerdo con la ciencia escolar las pueda transmitir a sus alumnos; y la segunda, relacionada con la anterior, tiene que ver con la formación de futuros profesores. Tradicionalmente se ha dicho que los graduados que se preparan para ser profesores tienen una excesiva formación en la materia en la cual se han graduado y adolecen de una formación en los aspectos pedagógicos, didácticos y de metodología para la enseñanza de dicha materia. Por ello, su formación se centra en estos últimos temas en detrimento del análisis didáctico del contenido correspondiente a los primeros. A la vista de lo encontrado en este trabajo parecería que no deberían descuidarse ninguno de los dos aspectos señalados, al menos en este campo conceptual.

Por último, cabe seńalar que, aunque existe gran cantidad de actividades de aula centradas en los dos aspectos seńalados, apenas se han encontrado estudios que investiguen metodologías de enseñanza y aprendizaje que abarquen de un modo global este tema, si se exceptúan los mencionados anteriormente en este trabajo.

Por todo lo expuesto, creemos necesario orientar las futuras investigaciones hacia el diseño, implementación y evaluación de propuestas de enseñanza innovadoras, eficaces y basadas en los resultados 
de la investigación educativa, que ayuden a superar las dificultades y cubrir las carencias mostradas en este trabajo, en un campo conceptual de tanta importancia desde el punto de vista social.

Tabla 3.

Coincidencias entre las ideas de alumnos y profesores en formación

\begin{tabular}{|c|c|}
\hline Conceptos & $\begin{array}{c}\text { Coincidencias entre las ideas de alumnos y } \\
\text { profesores en formación }\end{array}$ \\
\hline \multirow{2}{*}{$\begin{array}{l}\text { Sobre las fuentes } \\
\text { radiactivas }\end{array}$} & $\begin{array}{l}\text { Las fuentes radiactivas son concebidas como compuestas únicamente } \\
\text { por átomos radiactivos. }\end{array}$ \\
\hline & Las fuentes radiactivas son de origen humano (artificiales) \\
\hline \multirow{3}{*}{$\begin{array}{l}\text { Sobre los términos } \\
\text { irradiación y } \\
\text { contaminación }\end{array}$} & No diferenciación entre los términos irradiación y contaminación. \\
\hline & Un objeto expuesto a radiación se convierte en radiactivo. \\
\hline & $\begin{array}{l}\text { Ausencia o interpretación errónea del proceso de absorción de la } \\
\text { radiación. }\end{array}$ \\
\hline \multirow{4}{*}{$\begin{array}{c}\text { Sobre el } \\
\text { decaimiento } \\
\text { radiactivo y } \\
\text { período de } \\
\text { semidesintegración }\end{array}$} & Interpretación errónea a nivel atómico del proceso de decaimiento. \\
\hline & $\begin{array}{l}\text { Después de un tiempo igual a un período de semidesintegración } \\
\text { desaparece la mitad de la sustancia radiactiva. }\end{array}$ \\
\hline & La actividad permanece constante en el tiempo. \\
\hline & $\begin{array}{l}\text { Influyen factores externos al núcleo como la temperatura o el estado } \\
\text { físico. }\end{array}$ \\
\hline \multirow{2}{*}{$\begin{array}{l}\text { Sobre los efectos de } \\
\text { la radiactividad y } \\
\text { medidas protectoras }\end{array}$} & $\begin{array}{l}\text { La radiactividad o radiación ionizante es especialmente nociva para los } \\
\text { seres vivos. }\end{array}$ \\
\hline & $\begin{array}{l}\text { Como medidas protectoras se citan ejemplos del ámbito social (batas } \\
\text { de plomo, máscaras antigás, trajes blancos "anti-radiación”) y no se } \\
\text { tiene en cuenta el tipo de radiación. }\end{array}$ \\
\hline
\end{tabular}

\section{REFERENCIAS BIBLIOGRÁFICAS}

Al-Azmi, D., Karunakara, N. y Mustapha, A. O. (2013). Teaching about natural background radiation. Physics Education, 48(4), pp. 506-511.

http://dx.doi.org/10.1088/0031-9120/48/4/506

Al-Azmi, D., Mustapha A. O. y Karunakara N. (2012). Radon adsorbed in activated charcoal-a simple and safe radiation source for teaching practical radioactivity in schools and colleges. Physics Education, 47(4), pp. 471-475.

http://dx.doi.org/10.1088/0031-9120/47/4/471

Alsop, S. (2000). Meeting the needs of lifelong learners: recognizing a conative dimension in physics education. Physics Education, 35(3), pp. 202-208. http://dx.doi.org/10.1088/0031-9120/35/3/313

Ausop, S. (2001). Living with and learning about radioactivity: a comparative conceptual study. International Journal of Science Education, 23(3), pp. 263-281. http://dx.doi.org/10.1080/095006901750066510

Alsop, S. y WatTs, M. (1997). Sources from a Somerset village: A model for informal learning about radiation and radioactivity. Science Education, 81(6), pp. 633-650.

http://dx.doi.org/10.1002/(SICI) 1098-237X(199711)81:6<633::AID-SCE2>3.3.CO;2-1 http://dx.doi.org/10.1002/(SICI) 1098-237X(199711)81:6<633::AID-SCE2>3.0.CO;2-J

Alsop, S. y WatTs, M. (2000). Facts and feelings: exploring the affective domain in the learning of physics. Physics Education, 35(2), pp. 132-138.

http://dx.doi.org/10.1088/0031-9120/35/2/311 
Alsop, S., Watts, M. y Hanson, J. (1998). Pupils' perception of radiation and radioactivity: the wary meet the unsavoury. School Science Review, 79(289), pp. 75-79.

Anjos, R. M., Veiga, R., Carvalho, C., Sanches, N., Estellita, L., Zanuto, P. y Macario, K. (2008). Natural sources of radiation exposure and the teaching of radioecology. Physics Education, 43(4), pp. 423-427.

http://dx.doi.org/10.1088/0031-9120/43/4/012

Atwood, C. H. (1992). How Much Radon Is Too Much? Journal of Chemical Education, 69(5), pp. 351-355.

http://dx.doi.org/10.1021/ed069p351

Aubrecht, G. J. y Torick, D. A. (2000). Radioactivity: A Study of Student Ideas and Development of a Curriculum Based on the Findings. En M. A. Moreira (ed.). Proceedings of the Seventh Inter American Conference in Physics Education (pp. 16-32). Porto Alegre, Brasil: IAC.

Baker, A., Phelps, W., Alden, R., Hawkins, B. y McCauley, H. (1973). Introduction to Nucleonics: A Laboratory Course. Illinois: Crystal Lake, Ill. High School District 155.

Bindel, T. H. (1988). The Tasmanian Empire: A Radioactive Dating Activity. Journal of Chemical Education, 65(1), pp. 47-48.

http://dx.doi.org/10.1021/ed065p47

Boyes, E. y Stanisstreet, M. (1994). Children's Ideas about Radioactivity and Radiation: sources, mode of travel, uses and dangers. Research in Science \& Technological Education, 12(2), pp. 145-160. http://dx.doi.org/10.1080/0263514940120204

Brown, T. (2014). A Simple Example of Radioactive Dating. The Physics Teacher, 52, pp. 115-117. http://dx.doi.org/10.1119/1.4862120

Christian, M. C. (1988). An Exercise to Teach Concepts of Half-Life without using Radioactive Isotopes. Journal of Chemical Education, 65(1), pp. 48-49. http://dx.doi.org/10.1021/ed065p48

Colclough, N. D., Lock, R. y Soares, A. (2011). Pre-service Teachers' Subject Knowledge of and Attitudes about Radioactivity and Ionising Radiation. International Journal of Science Education, 33(3), pp. 423-446. http://dx.doi.org/10.1080/09500691003639905

Cookson, W. (1999). Detecting Radon Daughter Products using a Balloon. School Science Review, 81 (294), pp. 124-127.

Cooper, S., Yeo, S. y Zadnik, M. (2003). Australian students' views on nuclear issues: Does teaching alter prior beliefs? Physics Education, 38(2), pp. 123-129. http://dx.doi.org/10.1088/0031-9120/38/2/303

Couch, J. G. y Vaughn, K. L. (1995). Radioactive consumer products in the classroom. The Physics Teacher, 33(1), pp. 18-22.

http://dx.doi.org/10.1119/1.2344121

De Posada, J. M. y Prieto, T. (1990). Exploraciones gráficas de ideas extraescolares de los alumnos sobre radiactividad. Enseñanza de las Ciencias, 8(2), pp. 127-130.

Eujkelhof, H. M. C. (1989). Teaching about radioactivity and risk. En Proceedings of the International Conference on Energy Alternatives/Risk Education (vol. I), pp. 176-183. 7-13 de Septiembre en Lake Balaton (Hungary). Edited by George Marx, Department of Atomic Physics. Eötvös University, Budapest.

Eujkelhof, H. M. C., Klaasen, K. y Lijnse, P. L. (1989). Pupil's perception of radiation risks and its relevance for education. En Proceedings of the International Conference on Energy Alternatives/Risk Education (vol. I), pp. 170-175. 7-13 de septiembre en Lake Balaton (Hungary). Edited by George Marx, Department of Atomic Physics. Eötvös University, Budapest. 
Eijkelhof, H. M. C. y Lijnse, P. (1988). The role of research and development to improve STS education: experiences from the PLON project. International Journal of Science Education, 10(4), pp. 465-474. http://dx.doi.org/10.1080/0950069880100413

ErgüL, N. R. (2012). Determining the Knowledge Level of Pre-Service Teachers' on Radioactivity and Radiation. Educational Research and Reviews, 7(1), pp. 36-43. http://dx.doi.org/10.5897/ERR11.048

Esteban, S. y Pérez-Esteban, J. (2012). Estudiando el fenómeno de la radiactividad a través de noticias de prensa: el caso del espía ruso envenenado. Revista Eureka sobre Enseñanza y Divulgacion de las Ciencias, 9(2), pp. 294-306.

García-Carmona, A. y Criado, A. M. (2008). Enfoque CTS en la enseñanza de la energía nuclear: análisis de su tratamiento en textos de física y química de la ESO. Enseñanza de las Ciencias, 26(1), pp. 107-124.

Gipps, J. (1995). Potassium counts. School Science Review, 77(279), pp. 90-93.

Gutiérrez, E. E., Capuano, V. C., Perrota, M. T., De la Fuente, A. M., Follari, B. del R. (2000). ¿Qué piensan los jóvenes sobre radiactividad, estructura atómica y energía nuclear? Enseñanza de las Ciencias, 18(2), pp. 247-254.

Harrison, A. G. y Treagust, D. F. (1996). Secondary Students' Mental Models of Atoms and Molecules: Implications for Teaching Chemistry. Science Education, 80(5), pp. 509-534.

http://dx.doi.org/10.1002/(SICI)1098-237X(199609)80:5<509::AID-SCE2>3.0.CO;2-F

Hawkins, B. y Phelps, W. (1975). A course in nuclear radiation for all high school students. The Physics Teacher, 13(5), pp. 297-298.

http://dx.doi.org/10.1119/1.2339152

Henriksen, E. K. y Jorde, D. (2001). High School Students' Understanding of Radiation and the Environment: Can Museums Play a Role? Science Education, 85(2), pp. 189-206.

http://dx.doi.org/10.1002/1098-237X(200103)85:2<189::AID-SCE60>3.0.CO;2-S

Herrman, R. A. (1974). Concentrating radioactivity. Journal of Chemical Education, 51(6), pp. 420421.

http://dx.doi.org/10.1021/ed051p420

Hughes, E. A. y Zalts, A. (2000). Radioactivity in the Classroom. Journal of Chemical Education, 77(5), pp. 613-614.

http://dx.doi.org/10.1021/ed077p613

Hutchinson, S. G. y Hutchinson, F. I. (1997). Radioactivity in Everyday Life. Journal of Chemical Education, 74(5), pp. 501-505.

http://dx.doi.org/10.1021/ed074p501

Jesse, K. E. (2003). Computer Simulation of Radioactive Decay. The Physics Teacher, 41(9), pp. 542-543. http://dx.doi.org/10.1119/1.1631626

Jho, H., Yoon, H. G. y KIM, M. (2014). The relationship of science knowledge, attitude and decision making on socio-scientific issues: The case study of students' debates on a nuclear power plant in Korea. Science \& Education, 23(5), pp. 1131-1151.

http://dx.doi.org/10.1007/s11191-013-9652-z

Jona, K. y Vondracek, M. (2013). A Remote Radioactivity Experiment. The Physics Teacher, 51, pp. 25-27.

http://dx.doi.org/10.1119/1.4772033

Kolstø, S. D. (2001). Scientific Literacy for Citizenship: Tools for Dealing with the Science Dimension of Controversial Socioscientific Issues. Science Education, 85(3), pp. 291-310.

http://dx.doi.org/10.1002/sce.1011 
Kowalski, L. (1981). Simulating radioactive decay with dice. The Physics Teacher, 19(2), pp. 113-113. http://dx.doi.org/10.1119/1.2340711

Kuhn, J., Molz, A., Gröber, S. y Frübis, J. (2014). iRadioactivity-Possibilities and Limitations for Using Smartphones and Tablet PCs as Radioactive Counters. The Physics Teacher, 52(6), pp. 351356.

http://dx.doi.org/10.1119/1.4893089

Lapp, D. R. (2010). Obtaining and Investigating Unconventional Sources of Radioactivity. The Physics Teacher, 48(2), pp. 90-92. http://dx.doi.org/10.1119/1.3293653

Leenson, I. A. (2006). How Radioactive Are You? Journal of Chemical Education, 83(2), pp. 214-214. http://dx.doi.org/10.1021/ed083p214.1

Lemke, J. L. (1997). Aprender a hablar ciencia. Lenguaje, aprendizaje y valores. Barcelona: Paidós.

MaK, S. (1999). Radioactivity Experiments for Project Investigation. The Physics Teacher, 37, pp. 536539.

http://dx.doi.org/10.1119/1.880397

McGeachy, F. (1988). Radioactive Decay-An Analog. The Physics Teacher, 26(1), pp. 28-29. http://dx.doi.org/10.1119/1.2342411

Millar, R., Klaasen, K. y Eijkelhof, H. (1990). Teaching about Radiactivity and Ionising Radiation: an alternative approach. Physics Education, 25, pp. 338-342. http://dx.doi.org/10.1088/0031-9120/25/6/310

Millar, R. y Singh, J. (1996). School students' understanding of processes involving radioactive substances and ionizing radiation. Physics Education, 31(1), pp. 27-33.

http://dx.doi.org/10.1088/0031-9120/31/1/019

Myers, F. (2012). Temporary Personal Radioactivity. The Physics Teacher, 50, pp. 499-501. http://dx.doi.org/10.1119/1.4758157

NakiboĞLu, C. y Tekin, B. B. (2006). Identifying students' misconceptions about nuclear chemistry. Journal of Chemical Education, 83(11), pp. 1712-1718. http://dx.doi.org/10.1021/ed083p1712

Nusbaum, J. (1989). La constitución de la materia como conjunto de partículas en la fase gaseosa. En Driver, Guesne y Tiberghien (Eds.). Ideas cientificas en la infancia y la adolescencia (pp. 196-224). Madrid: Morata y MEC.

Peplow, D. E. (1999). Fiestaware ${ }^{\mathrm{TM}}$ Radiography. The Physics Teacher, 37(5), pp. 316-318. http://dx.doi.org/10.1119/1.880278

Pozo, J. I. (1996). La psicología cognitiva y la educación científica. Investigaçôes em Ensino de Ciências, 1(2), pp. 110-131.

Prather, E. (2005). Students' beliefs about the role of atoms in radioactive decay and half-life. Journal of Geoscience Education, 53(4), pp. 345-354.

Prather, E. E. y Harrington, R. R. (2001). Student Understanding of Ionizing Radiation and Radioactivity. Journal of College Science Teaching, 31(2), pp. 89-93.

Ronneau, C. (1990). Radioactivity: A Natural Phenomenon. Journal of Chemical Education, 67(9), pp. 736-737. http://dx.doi.org/10.1021/ed067p736

Russo, R. N. (1999). La Fiesta Radioactiva. Distinguishing Alpha, Beta and Gamma Emissions from Orange-Glaze Dinnerware. Journal of College Science Teaching, 28(5), pp. 348-351.

Schultz, E. (1997). Dice-Shaking as an Analogy for Radioactive Decay and first-order Kinetics, Journal of Chemical Education, 74(5), pp. 505-507.

http://dx.doi.org/10.1021/ed074p505 
Showers, D. E. y Shrigley, R. L. (1995). Effects of knowledge and persuasion on high冈school students' attitudes toward nuclear power plants. Journal of Research in Science Teaching, 32(1), pp. 29-43. http://dx.doi.org/10.1002/tea.3660320105

UNSCEAR (2010). Sources and effects of ionizing radiation. United Nations Scientific Committee on the Effects of Atomic Radiation. Report to the General Assembly with Scientific Annexes, 2008. New York: Unites Nations Publications. ISBN 978-92-1-142274-0.

Whitcher, R. (2011). Practical work using low-level radioactive materials available to the public. School Science Review, 92(341), pp. 65-74.

Wu, Y. T. y Tsai, C. C. (2007). High school students' informal reasoning on a socio-scientific issue: Qualitative and quantitative analyses. International Journal of Science Education, 29(9), pp. 1.1631.187 . http://dx.doi.org/10.1080/09500690601083375

Zeidler, D. L., Sadler, T. D., Simmons, M. L. y Howes, E. V. (2005). Beyond STS: A researchbased framework for socioscientific issues education. Science Education, 89(3), pp. 357-377. http://dx.doi.org/10.1002/sce.20048

\section{REFERENCIAS PROCEDENTES DE PÁGINAS WEB:}

Eujkelhof, H. M. C. (1990). Radiation and Risk in Physics Education. Utrecht, University of Utrecht. Disponible en línea: <http://www.iaea.org/inis/collection/NCLCollectionStore/_Public /22/010/22010294.pdf>. Último acceso, 24/11/2014.

FEndT,W.AppletsJavadeFísica.Disponibleenlínea:<http://www.walter-fendt.de/ph6es/lawdecay_es.htm>. Último acceso, 16/09/2015.

Franco, A. Física con Ordenador: Curso interactivo de Física en internet. Disponible en línea: <http:// www.sc.ehu.es/sbweb/fisica/cuantica/desintegracion/radio.htm\#Actividades>. Último acceso, 16/09/2015. 
ANEXO 1.

Bases de datos en las que se ha localizado la información

\begin{tabular}{|c|c|c|}
\hline Base de datos & Características & Dirección \\
\hline Dialnet & $\begin{array}{l}\text { Índices de revistas científicas y } \\
\text { humanísticas de España } \\
\text { Latinoamé-rica, monografías, tesis } \\
\text { doctorales... }\end{array}$ & $\begin{array}{l}\text { Universidad de la Rioja } \\
\text { http://dialnet.unirioja.es/ }\end{array}$ \\
\hline Google académico & $\begin{array}{lll}\begin{array}{l}\text { Buscador } \\
\text { especializada }\end{array} & \text { de } & \text { bibliografía } \\
\end{array}$ & http://scholar.google.es/ \\
\hline $\begin{array}{l}\text { Biblioteca de Educa- } \\
\text { ción }\end{array}$ & Sumarios de revistas & $\begin{array}{l}\text { M.E.C. y D. } \\
\underline{\text { http://www.mecd.gob.es/bibliotec }} \\
\underline{\text { a-central/sumario-revistas.html }}\end{array}$ \\
\hline British Education Index & $\begin{array}{l}\text { Revistas británicas relacionadas con } \\
\text { la educación. Permite la búsqueda } \\
\text { combinada en las fuentes Education- } \\
\text { Line, Internet resources y Journal } \\
\text { articles. Fundamentalmente } \\
\text { documentos de libre acceso. }\end{array}$ & $\begin{array}{l}\text { http://www.leeds.ac.uk/bei/index. } \\
\text { html }\end{array}$ \\
\hline $\begin{array}{l}\text { The Educator's Refe- } \\
\text { rence Desk }\end{array}$ & $\begin{array}{l}\text { Recopila numerosos enlaces a } \\
\text { diversos recursos educativos y } \\
\text { cuenta con una colección de más de } \\
2000 \text { unidades didácticas para } \\
\text { distintas áreas y niveles de } \\
\text { enseñanza. }\end{array}$ & $\begin{array}{l}\text { Servicio de } \text { Informacion del } \\
\text { Instituto de Syiracusa } \\
\text { (NuevaYork) } \\
\text { http://www3.eduref.org/ }\end{array}$ \\
\hline $\begin{array}{lr}\text { ERIC. } & \text { Education } \\
\text { Resources } & \text { Information } \\
\text { Center } & \end{array}$ & $\begin{array}{l}\text { Registros bibliográficos de artículos } \\
\text { de revistas y otros materiales } \\
\text { relacionados con la educación. } \\
\text { Desde algunos registros es posible } \\
\text { acceder al texto completo del } \\
\text { documento. }\end{array}$ & $\begin{array}{l}\text { Education Resources Information } \\
\text { Center (ERIC) dependiente del } \\
\text { gobierno de Estados Unidos. } \\
\text { http://eric.ed.gov/ }\end{array}$ \\
\hline Intute & $\begin{array}{l}\text { Recursos sobre diversos campos, } \\
\text { entre los que se encuentra la } \\
\text { educación. Servicio gratuito. }\end{array}$ & $\begin{array}{l}\text { Recurso sostenido por un } \\
\text { consorcio de varias universidades } \\
\text { británicas. } \\
\text { http://www.mecd.gob.es/bibliotec } \\
\text { a-central/recursos- } \\
\text { electronicos/bases-datos.html }\end{array}$ \\
\hline Latindex & $\begin{array}{l}\text { Cuenta con tres bases de datos: } \\
\text { Directorio, con datos de todas las } \\
\text { revistas registradas; Catálogo, que } \\
\text { incluye únicamente las revistas que } \\
\text { cumplen los criterios de calidad } \\
\text { editorial diseñados por Latindex; y } \\
\text { Enlace a revistas electrónicas, que } \\
\text { permite el acceso a los textos } \\
\text { completos cuando se encuentran } \\
\text { disponibles. }\end{array}$ & $\begin{array}{l}\text { Información sobre las revistas de } \\
\text { investigación y de divulgación } \\
\text { científica y cultural que se editan } \\
\text { en los países de América Latina, } \\
\text { el Caribe, España y Portugal, } \\
\text { tanto en papel como en formato } \\
\text { electrónico. } \\
\text { http://www.latindex.unam.mx/ }\end{array}$ \\
\hline
\end{tabular}


ANEXO 2.

Índices de impacto de las revistas en las que están publicados los artículos seleccionados

\begin{tabular}{|c|c|}
\hline Revista & Datos \\
\hline Educational Research and Reviews & $\begin{array}{l}\text { Editada por Academic Journals, Nigeria. } \\
\text { Índice de impacto SJR (2013): 0,150; Q4 } \\
\text { SIDI ICDS (2013): } 7,345\end{array}$ \\
\hline Enseñanza de las Ciencias & $\begin{array}{l}\text { Editada por el ICE de la Universidad Autónoma de Barcelona y la } \\
\text { Univ. de Valencia, España. } \\
\text { Índice de impacto en JCR (2013): 0,238; Q4. } \\
\text { Índice de impacto SJR (2013): 0,103; Q4. } \\
\text { Índice de Impacto IN-RECS (2011): 0,194; Q1 } \\
\text { SIDI ICDS (2013): } 7.977\end{array}$ \\
\hline $\begin{array}{llll}\text { International Journal of } & \text { Science } \\
\text { Education } & & & \end{array}$ & $\begin{array}{l}\text { Editada por Routledge, Reino Unido. } \\
\text { Índice de Impacto JCR (2013): 1,516; Q1 } \\
\text { Índice de impacto SJR (2013): 1,656; Q1 } \\
\text { SIDI ICDS (2013): 9,977 }\end{array}$ \\
\hline Journal of Chemical Education & $\begin{array}{l}\text { Editada por American Chemical Society, EEUU. } \\
\text { Índice de Impacto JCR (2013): 1,001; Q4 (JCR Science Edition, } \\
\text { Chemistry multidisciplinary) } \\
\text { Índice de impacto SJR (2013): 0,332; Q3 } \\
\text { SIDI ICDS (2013): 9,977 }\end{array}$ \\
\hline Journal of College Science Teaching & $\begin{array}{l}\text { Editado por la NSTA, EEUU. } \\
\text { SIDI ICDS (2013): } 6,477\end{array}$ \\
\hline Journal of Geoscience Education & $\begin{array}{l}\text { Editada por la Association of Geoscience Teachers (NAGT), EEUU. } \\
\text { Índice de impacto SJR (2013): 0,236; Q3 } \\
\text { SIDI ICDS (2013): } 7,977\end{array}$ \\
\hline Physics Education & $\begin{array}{l}\text { Editada por IOP Science, Reino Unido. } \\
\text { Índice de impacto SJR (2013): 0,230; Q3 } \\
\text { SIDI ICDS (2013): 9,977 }\end{array}$ \\
\hline $\begin{array}{l}\text { Research in Science \& Technological } \\
\text { Education }\end{array}$ & $\begin{array}{l}\text { Editada por Routledge, Reino Unido. } \\
\text { Índice de impacto en JCR (2012): 0,500; Q2. } \\
\text { Índice de impacto SJR (2013): 0,403; Q2 } \\
\text { SIDI ICDS (2013): 9,977 }\end{array}$ \\
\hline Revista de educación & $\begin{array}{l}\text { Editada por el Minist. Educación y Ciencia, Inst. Nacional de Calidad } \\
\text { y Evaluación, España. } \\
\text { Îndice de impacto JCR (2012): 0,309, Q4 } \\
\text { Índice de impacto SJR (2013): 214, Q3 } \\
\text { Índice de Impacto IN-RECS (2011) 0.587, Q1 } \\
\text { SIDI ICDS (2013): 9,977 }\end{array}$ \\
\hline $\begin{array}{l}\text { Revista Eureka sobre Enseñanza y } \\
\text { Divulgación de las Ciencias }\end{array}$ & $\begin{array}{l}\text { Editada por la Universidad de Cádiz y APAC-EUREKA. España. } \\
\text { Índice de Impacto IN-RECS (2011) 0,092, Q2 } \\
\text { SIDI ICDS (2013): 5,954 }\end{array}$ \\
\hline Revista Iberoamericana de Educación & $\begin{array}{l}\text { Editada por la Organización de Estados Iberoamericanos (OEI), } \\
\text { España. } \\
\text { Índice de Impacto IN-RECS (2011): 0.156, Q2 } \\
\text { SIDI ICDS (2013): 6,301 }\end{array}$ \\
\hline School Science Review & $\begin{array}{l}\text { Editada por The Association for Science Education, Reino Unido. } \\
\text { SIDI ICDS (2013): } 4,477\end{array}$ \\
\hline Science Education & $\begin{array}{l}\text { Editada por Wiley, EEUU } \\
\text { Índice de Impacto JCR (2013): 2,921; Q1. } \\
\text { Índice de impacto SJR (2013): 4,476; Q1 } \\
\text { SIDI ICDS (2013): 9,977 }\end{array}$ \\
\hline The Physics Teacher & $\begin{array}{l}\text { Editada por American Association of Physics Teachers, EEUU } \\
\text { SIDI, ICDS: } 6,477\end{array}$ \\
\hline
\end{tabular}




\title{
Current issues in teaching and learning radioactivity in secondary education
}

\author{
Javier Corbelle Cao, José Manuel Domínguez Castiñeiras \\ Departamento de Didáctica de las Ciencias Experimentales. Universidad de Santiago de Compostela. España \\ jcorbelle@edu.xunta.es, josemanuel.dominguez@usc.es
}

The objective of this work is to understand the state of didactic research about the different issues on teaching and learning in the conceptual field of radioactivity. In order to do this, a wide bibliographical review is undertaken using a suitable database mentioned in the article (Annex 1). Also, the studies found here have been selected employing the most common impact factors (See table 1 and annex 2 in the article).

After a preliminary examination of the selected works, it was decided to analyze them from three distinct perspectives:

a) Students' ideas.

b) Pre-service teachers' ideas.

c) Classroom activities and teaching proposals.

The analysis of the ideas of students and pre-service teachers was carried out using two perspectives: macroscopic and atomic-nuclear, establishing, for each perspective, the following categories.

Students' ideas

- Macroscopic perspective: about the terms radioactivity/radioactive material/radiation; about radioactive sources; about the terms irradiation/contamination; about the ways the emitted radiation travels; about the effects of radioactivity and protective actions.

- Atomic-nuclear perspective: about the radioactive atom; about the radioactive decay and half-life.

Ideas of pre-service teachers

- Macroscopic perspective: about radioactive sources; about the terms irradiation/contamination; about the effects of radioactivity and protective actions.

- Atomic-nuclear perspective: about the radiation-matter interaction; about the radioactive decay and half-life.

It was found that students as well as pre-service teachers hold a great number of misconceptions, sharing some of them. For example, the students make an undifferentiated use of the terms «radioactivity», «radioactive material», and «radiation». They are also unaware of the short range of the radiation emitted by radioactive substances and the great distances those substances can travel.

Both students and pre-service teachers associate radioactivity with human action, being unaware of our continued exposure to natural background radiation. They also consider that an object that has been irradiated becomes radioactive, or that it absorbs the radiation received, accumulating it inside as if it was a pollutant. Similarly, they claim that the radiation emitted by a radioactive substance may be altered modifying its temperature or physical condition. Radioactivity is always associated with danger without taking into account the concept of dose. When citing protective measures, the type of radiation ( $\mathrm{a}, \mathrm{b}$ o g) is not considered. Instead, examples from social experience, such as visits to the hospital or television programmes (e.g. anti-radiation white lab coats, or anti-gas masks), are mentioned.

From an atomic-nuclear perspective, students make use of factors outside the atomic core such as valence electrons to justify that an atom is radioactive. Both students and pre-service teachers believe that radioactive sources are made up only of radioactive atoms. They also believe that the radioactive substance simply disappears after a half-life period.

In relation to section $\mathrm{C}$, numerous classroom activities have been found to focus mainly on two aspects: activities that aim to highlight the natural background radiation and activities that illustrate the process of radioactive decay. The advantages offered from the educational point of view are analyzed, but the risk of reinforcing the aforementioned misconceptions is also noted. This section also includes works dealing with radioactivity from the point of view of the Science, Technology and Society and also as a controversial socio-scientific issue. The aspect usually dealt with regarding this is nuclear energy as a means of electrical energy production and the problem associated with the radioactive waste. There also are some studies that deal with learning about radioactivity in non-formal contexts. However, almost no works have been found that deal with teaching and learning methodologies about this topic. For this reason, it is suggested to guide future research towards the design, implementation and evaluation of innovative teaching proposals based on the results of the educational research that helps to overcome difficulties and fills in the gaps shown in this work. 